\title{
Is there still a place for the cftmea (french classification of mental disorders in children and adolescents) in the child and adolescent psychiatry of the xxist century ?
}

Bruno Falissard 1,

1 Université Paris-Sud,

\section{Abstract}

Background:

During decades the DSM has been de facto the unique classification of mental disorders in children and adolescents (the ICD appearing in many aspects as a clone of the DSM). In this first part of the XXIst century the situation appears more complex, in particular since the apparition of the RDoC (Research Domain Criteria).

Methods:

opinion paper

Results:

The paper presents rapidly 6 classifications of mental disorders that can be used in child and adolescent psychiatry: CCTM (Chinese classification), DSM, ICD, RDoC, PDM and CFTMEA. It discusses the interests and limits of the CFTMEA, a classification based on a psychodynamic conceptualization of mental disorders, in which the notions of neurosis, psychosis and borderline structure are central.

\section{Conclusion:}

DSM was presented as theoretically neutral. There is now a need to provide classifications of mental diseases that have a strong theoretical background, whatever this background is (biological, psychodynamic, etc.)

Keywors:

DSM, ICD, PDM, CFTMEA, RDoC, diagnosis, classifications, mental disorders, psychoanalysis

\section{IACAPAP ArXiv}


Quelle place pour la CFTMEA dans la psychiatrie du XXlème siècle ?

Il existe aujourd'hui 5 systèmes de classification des maladies mentales. La CCTM (classification chinoise des troubles mentaux), non totalement traduite, est sûrement la moins connue de toutes. Proche du DSM et de la CIM, elle contient également des entités cliniques originales, comme le koro, syndrome de rétraction des organes génitaux.

A l'opposé, le DSM américain est très largement utilisé dans la plupart des pays. Cette classification est l'objet de tous les fantasmes. Validée scientifiquement ? A-théorique ? Conçue pour la recherche ? Disons plutôt qu'elle a fait l'objet d'un nombre considérable d'études qui en étayent plus ou moins la structure; qu'elle repose sur une logique syndromique/sémiologique ; qu'elle a permis l'éclosion de l'épidémiologie psychiatrique, mais qu'elle est également utilisée aujourd'hui pour classer des patients en vue du remboursement de leurs soins, ce qui est loin d'être anodin. Enfin, reconnaissons aux américains la grande précision de leurs descriptions sémiologiques : le DSM est un instrument qui gagne à être lu. Il donne une excellente idée de la partie la plus visible des tableaux cliniques.

La classification internationale des maladies (CIM) de l'OMS n'est que faussement internationale. Elle est en réalité un clone du DSM. Cela s'explique par la contrainte politique forte que subi l'OMS : la nécessité de faire consensus. Or la puissance des associations américaines de psychiatrie fait que le consensus international se doit d'être avant tout compatible avec le DSM : c'est d'ailleurs explicite dans l'introduction de la toute nouvelle CIM 11.

Le monde des classifications psychiatrique a vécu une révolution au début des années 2010. Cette révolution est venue des Etats-Unis et, de façon assez paradoxale, a trouvé son origine dans une critique virulente du DSM. Pour le NIMH (National Institute of Mental Health), l'absence de progrès constaté dans les 20 dernières années dans le domaine des thérapeutiques psychiatriques viendrait du fait que le DSM est purement clinique et déconnecté des connaissances accumulées par les 
neurosciences. Dans les autres spécialités médicales, en particulier dans le domaine du cancer ou des maladies inflammatoires, c'est un recours intensif aux recherches en biologie moléculaire qui a permis l'avènement de thérapies ciblées considérées par beaucoup comme de véritables ruptures. Si l'on veut que la thérapeutique psychiatrique progresse de la même façon, il est alors suggéré par le NIMH que la nosographie psychiatrique repose sur les données issues des neurosciences fondamentales. Les RDoc (Research Domain Criteria) obéissent à cette logique. Ils regroupent un petit nombre de syndromes ou plutôt de domaines caractérisés par des corrélats génétiques, des caractéristiques neurobiologiques, un schéma d'expressions comportementales, un ensemble de scores psychométriques, etc. II n'y a plus d'opposition entre le normal et la pathologique mais un continuum d'expression phéno/génotypique. Les RDoc sont clairement développés pour la recherche fondamentale. Ils peuvent faire réfléchir le clinicien, mais sont de peu d'utilité dans la relation médecin malade, ça n'est clairement pas leur objectif.

La situation est exactement à l’opposé pour le PDM-2 (Psychodynamic Diagnosis Manual). Ce long document de plus de 1000 pages est un traité de psychopathologie visant à aider le clinicien à se représenter son patient dans le but d'établir une relation thérapeutique. Le PDM-2 propose plusieurs axes descriptifs. Le premier axe considère le patient sous l'angle de sa structure psychopathologique : psychotique, limite, névrotique. Le second axe s'attache à décrire les facettes les plus saillantes du fonctionnement psychologique du patient (sous la forme de mécanismes de coping, de défenses, etc.). Le troisième axe, phénoménologique, est calé sur le DSM et explicite le vécu subjectif des patients relevant de telle ou telle catégorie diagnostique.

Quelle est la place de la CFTMEA dans ce panorama pour le moins hétérogène ? Comme le PDM-2 et contrairement au DSM et à la CIM, la CFTMEA revendique un assise théorique forte, psychanalytique. Contrairement au PDM-2, mais au même titre que le DSM et la CIM, la CFTMEA n'est pas un manuel de psychopathologie mais un authentique système classificatoire. Quant aux RDoC, comme la CFTMEA ils s'inscrivent dans un champ théorique (neuroscientifique versus psychanalytique), mais 
les RDoC proposent une représentation multidimensionnelle utile pour la recherche, alors que la CFTMEA conduit à des catégories utilisables en clinique, par exemple pour aider les praticiens à se construire une représentation synthétique de leurs patients, pour faciliter leurs échanges avec les autres soignants ou avec les familles. La CFTMEA peut être également utilisée en épidémiologie (une étude a d'ailleurs été réalisée dans ce domaine (1)).

La CFTMEA a ses forces et ses faiblesses.

Parmi les forces, il y a bien sûr une structure qui, à l’opposé du patchwork nosographique que constitue le DSM, s'organise autour de lignes forces. Le socle classificatoire de la CFTMEA repose en effet sur les trois grandes catégories qui organisent depuis de nombreuses décennies la pensée psychopathologique : psychoses, états limites, névroses. Il est d'ailleurs curieux de lire régulièrement que cette tripartition ne dispose d'aucun élément de validité métrologique. Pas moins de deux articles publiés dans le Journal of Personality Disorders ont étudié la reproductibilité du Kernberg structural interview (1-2). Les deux trouvent des résultats voisins, avec des coefficients kappa autour de $0,5-0,6$, très similaires à ce que l'on retrouve pour la concordance diagnostique des instruments structurés relevant du DSM.

La CFTMEA dispose par ailleurs de catégories originales, particulièrement utiles dans la pratique clinique quotidienne, que I'on ne retrouve pas dans les classifications comme le DSM ou la CIM. On pensera notamment aux diagnostics de dysharmonie psychotique ou de dysharmonie évolutive, que le DSM a bien du mal à capturer malgré de nombreuses tentatives, certaines anciennes comme le MCDD (Multiple Complex Developmental Disorder), d'autre plus récente comme le DMDD (Disruptive Mood Dysregulation Disorder).

Enfin, la CFTMEA est la seule classification à proposer des labels qui sortent explicitement du champ de la pathologie puisqu'ils relèvent de « variantes de la normale ». Certains de nos patients nous sont en effet amenés pour des raisons qui ne relèvent pas de la psychiatrie. Les parents attendant souvent que l'on mette des mots sur leurs interrogations, il est utile de pouvoir dire « votre enfant présente 
simplement un 'Aspect original de la personnalité', mais ce n'est pas un diagnostic, une maladie, c'est une simple variante de la normale ».

La CFTMEA a bien sûr des faiblesses. Sa très faible utilisation en est une incontestablement. Cette faible utilisation est sûrement liée pour partie à sa nature francophone, mais pas uniquement. Les descriptions cliniques permettant de poser les diagnostics sont souvent trop elliptiques, faisant appel implicitement à des textes beaucoup plus complets publiés par ailleurs. Ainsi, le diagnostic de dysharmonie évolutive est bien difficile à poser pour qui se contente des seules explications proposées dans le manuel de classification.

Au total, les années 2020 vont vivre une véritable effervescence nosographique. Le DSM est de plus en plus critiqué, y compris dans ses propres rangs. Les classifications revendiquant une forte charge théorique ont maintenant droit de citer et se révèlent même comme des alternatives porteuses de progrès potentiels. Dans un tel contexte, à un moment ou beaucoup de cliniciens souhaitent penser la psychiatrie sous des angles pluriels, la CFTMEA a à l'évidence de beaux jours devant elles, surtout si nous la faisons vivre dans nos pratiques et dans nos enseignements.

(1) Quemada N. (1990) L'utilisation de la classification française des troubles mentaux de l'enfant et de l'adolescent dans une enquête nationale. Neuropsychiatrie de l'enfance et de l'adolescence. 10-12, 540-546.

(2) Theo J. M. Ingenhoven, Hugo J. Duivenvoorden, Janneke Brogtrop, Anne Lindenborn, Wim van den Brink, and Jan Passchier (2009). Brief Communications: Interrater Reliability for Kernberg's Structural Interview for Assessing Personality Organization. Journal of Personality Disorders: Vol. 23, No. 5, pp. 528-534.

(3) Jan J. Derksen, Jacobus W. Hummelen, and Piet J. Bouwens (1994). Interrater Reliability of the Structural Interview. Journal of Personality Disorders: Vol. 8, No. 2, pp. 131-139. 